\title{
The helping hand: How to bias kinesthetic aftereffects by instructional set*
}

\author{
DANIEL J. WEINTRAUB $\dagger$ and ANITA CAPLAN \\ University of Michigan, Ann Arbor, Michigan 41804
}

\begin{abstract}
After rubbing a block of a different size, Ss judged either the objective width of the test block or its felt width. With no preliminary information, judgments of objective and phenomenal width were equivalent. Judgments of objective but not of phenomenal width moved in the direction suggested by a truthful verbal briefing. (Prior practice with a visual display, the Mueller-Lyer arrowhead illusion presented as an analogy to the kinesthetic aftereffect task, proved to be ineffective.) Misinformation did not alter subsequent judgments. In no case was the measured aftereffect abolished or reversed. It is suggested that knowledge about the task modifies judgments rather than sensory experience.
\end{abstract}

The kinesthetic aftereffect (KAE) is produced by rubbing an inducing (I) block between the fingers. The induction procedure changes the subsequent judgment of the width of a test $(\mathrm{T})$ block. Width contrast is the aftereffect: the $T$ block feels narrower with I wider than $T$, or wider with I narrower than $T$. Previous research on kinesthetic aftereffects has been hampered by large variances, too little of which can be ascribed to independent variables. For those who have sought neurophysiological explanations, the large differences among individuals are both a bother and an embarrassment. For those who have pursued the concomitants of individual differences, the anemic test-retest reliabilities (Becker, 1960) are a severe handicap. A reasonable hypothesis is that instructions to Ss concerning their task in a KAE experiment permit a certain latitude in interpretation and execution. Ss' inferences and knowledge about the direction of the aftereffects, judgmental strategies adopted during testing, etc., can, at least in principle, be brought under experimental control by means of differential instructional set, thereby accounting for hitherto unexplained variability in KAE performance.

Wertheimer and Sheets (1968) provided a prior briefing concerning KAE, giving Ss practice with the Mueller-Lyer arrowhead illusion presented visually and pointing out by analogy the direction of kinesthetic aftereffects. Then, half of the group of Ss received physicalistic-set instructions: "When you know the effect of the rubbing you can compensate for it as you did with the Mueller-Lyer, and report how wide the standard bar actually is. Try to compensate for the effect of the rubbing and report how wide the bar actually is." The remainder received phenomenological-set instructions to "report how wide the standard bar feels. Please do not try to compensate

*Support was provided by a United States Public Health Service Research Scientist Development Award (K2-MH-35,253) to Daniel J. Weintraub. Research funds were provided by National Science Foundation Grant GB 8181.

†Address: Human Performance Center, Perry Building, 330 Packard Road, Ann Arbor, Michigan 48104. in any way for the effect of rubbing. Pay attention to and report how wide it feels." Wertheimer and Sheets (1968) found that, while the physicalistic set reduced the measured amount of aftereffect by more than half, a statistically significant aftereffect remained.

Enveloping the immediate question of the efficacy of instructional set is the more basic epistemological issue. We accept the proposition that we are aware of our nerves and not of objects. If that be the case, and if it is assumed that no visual or other cues are inadvertently divulged, then a perceiver's kinesthetic impressions are his link with the block, all that he can know "directly" about its width. If rubbing alters the information subsequently carried by the sensory system, then real-world blocks are perceived differently. Can the perceiver discern the effects of induction (rubbing)? Not by sense impression alone, certainly. However, if a perceiver can believe that he has felt the same block both before and after rubbing, and can remember the prior felt width, then he might correctly deduce the nature of the aftereffect. There are also tests based upon self-produced stimulation that a perceiver might apply. For example, after induction, he might touch fingers together (known zero width) and recognize the anomaly that fingers together seem a different distance apart when compared with memories from the preinduction state. The point is that in the total absence of sensory information other than kinesthetic cues, and even in the absence of prior "cognitive" information (reading about or being told about KAE), it is not impossible for a perceiver to predict correctly the effects of rubbing. (Such a feat would require, however, remembered kinesthetic information.) We may be able to transcend our own immediate sensory input to predict the world reasonably accurately. The question of whether prior information has changed how a perceiver experiences the world, or merely what he is willing to predict about it, seems amenable to experimental attack.

The approach used was to expand the Wertheimer-Sheets (1968) experiment. (Two of the experimental groups closely replicated the entire Wertheimer-Sheets experiment, except for a change in 


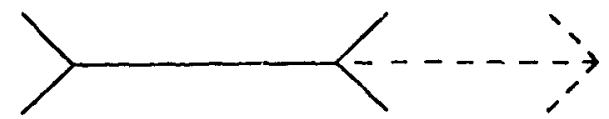

TRUTH DISPLAY

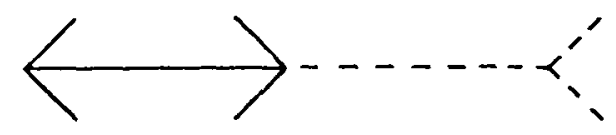

FALSEHOOD DISPLAY

Fig. 1. The two visual Mueller-Lyer practice displays used to reinforce verbal descriptions of the directionality of kinesthetic aftereffects. (Dotted lines depict movable portions of each display. All lines were actually solid.)

block widths to conform to the KAE apparatus at hand.) As before, half the total number of Ss received a physicalistic instructional set (judge how the width actually is), and the other half received a phenomenological instructional set (judge how the width feels). The major innovation was to manipulate the nature of the instruction prior to the KAE task. For some Ss, no prior instruction was provided. For others, true information about the effect of rubbing was provided (like Wertheimer \& Sheets, 1968). For others, misinformation concerning the effect of rubbing on judgments was provided, describing the outcome as the opposite of the real effect. In addition, some groups of Ss received only verbal descriptions, while others received verbal descriptions reinforced by practice with the Mueller-Lyer arrowhead display. The Mueller-Lyer practice was designed to match the verbal information and could be used to mislead Ss as necessary.

\section{METHOD}

Modifying the Wertheimer-Sheets experiment in order to misinform $S s$ about the directional change in judgments after rubbing was casy because the experiment, although correctly stating the directional change. was based upon a compound aftereffect. One hand rubbed an inducing block larger than the test block, while the other hand rubbed an I smaller than $T$. Thus, after induction, the width contrast that occurred was underestimation for one hand and simultaneously, overestimation for the other. When Wertheimer and Sheets told their Ss that the "overall effect is to cause you to overestimate the width of the standard bar [test block]." the orerall effect would in fact have been underestimation if the test block had been grasped by the other hand.

It is a simple matter, using the compound form of the Mueller-Lyer illusion (see Fig. 1), to reinforce visually either the correct or incorrect overall effect to be expected with KAE. because length overestimation (induced by inward-pointing arrowheads) and length underestimation (induced by outward-pointing arrowheads) occur simultaneously in each visual display, just as width overestimation and underestimation occur simultaneously in the KAE task.

\section{Subjects}

Thers were 192 right-handed male students at the Lniversity of Michigan who participated. Each was assigned randomly to one of eight groups, yielding $24 \mathrm{Ss}$ per group. The Ss were tested individually; each served only once.

\section{Apparatus}

The $I$ and $T$ blocks were 6 in. $(15.24 \mathrm{~cm})$ long and $1 \mathrm{in}$. $(2.54 \mathrm{~cm})$ deep. Each had parallel sides 2 in. $(5.08 \mathrm{~cm})$ in width for $T$ and $1 / 2$ in. $(1.27 \mathrm{~cm})$ and $3 \mathrm{in} .(7.62 \mathrm{~cm})$ in width for the $I$ blocks. A wedge, 25 in. $(63.5 \mathrm{~cm})$ long and 1 in. $(2.54 \mathrm{~cm})$ deep, had sides tapering from $3 \frac{1}{2} \mathrm{in} .(8.89 \mathrm{~cm})$ wide at the front to $1 / 2$ in. $(1.27 \mathrm{~cm})$ at the rear. Mounted lengthwise atop each block and the wedge was a rail supporting a sliding finger guide. To rub or judge, $S$ kept thumb and index finger in the slots of the $\mathrm{H}$-shaped guide while maintaining fingertip contact with block or wedge. The pieces of KAE apparatus rested on two platforms between which $S$ stood. The items of apparatus were supported above the platforms on columns. Each item was oriented with its longest axis parallel to the floor and pointing straight ahead with respect to $\mathrm{S}$. The wedge had its wide end forward. The items were concealed except during testing. when $S$ wore a large cardboard collar that prevented his seeing the equipment. The $T$ block was at S's left side, the wedge at his right. When S took a step back. the narrow I was at his left side and the wide I was at his right. The judging procedure followed that of Wertheimer and Sheets, with four pre- and four postinduction judgments (starting position on the wedge counterbalanced, etc.). For each judgment, $S$ was required to find the width on the wedge with his right hand that matched the width of $T$ in his left hand. After the preinduction judgments, $S$ stepped back and rubbed back and forth on the I blocks continuously for $1 \mathrm{~min}$. He then stepped forward for the postinduction judgments on T. Data are reported in centimeters of judged width for a match with the wedge.

All Ss were tested using the KAE procedure described above. In addition, some groups were given prior practice with a visual Mueller-Lyer arrowhead display. Two different displays were constructed (see Fig. 1). The dimensions of each, and the finish, solid white lines on a black background. match the display of Wertheimer and Sheets. The dotted portions of the displays in Fig. 1 depict the portions attached to a horizontal slide, which could be moved in or out to expose more or less of the horizontal line to the right of the center arrowhead. Note that the adjustable portions of these displays are on the right. as they were for an $\mathrm{S}$. the same side as the wedge on which he made subsequent KAE width adjustments. The use of a visual display followed the Wertheimer-Sheets procedure. The $\mathrm{E}$ manipulated the slide in accordance with S's instruction for four trials under phenomenologically' oriented instructions to determine when the line between inward-pointing arrowheads appeared just as long as the line between outward-pointing arrowheads. The data were then examined and discussed with $S$. the direction of effeets noted, etc. The $S$ was then informed that he should try to compensate for the illusion in the next four judgments (physicalistic set). All eight judgments were examined and discussed. Most Ss had produced a marked shift toward veridicality in their performance when trying to compensate for the illusion. Those who shifted only a little were also shown the data from Ss with large changes. In Fig. 1. the displays are labeled "falsehood display" and "truth display." but in neither case was misinformation provided about the Mueller-Lyer illusion. The labels refer to the way each display was used.

The net effect of the Wertheimer-Sheets KAE procedure is to lead $S$ to make a width judgment that is too wide, i.e.. an overestimate along the wedge with his right hand. Correct information discussing the net overestimation in KAE was reinforced by means of the Mueller-Lyer truth display. which induces $S s$ to expose too much horizontal line on the right. Misinformation about $\mathrm{K} A \mathrm{E}$ direction was given added emphasis by the falsehood display. Which indures $S$ s to expose too little horizontal line on the right. The analogy between Mueller-Lyer 
Table 1

Results of the KAE Experiment (Data in Centimeters)

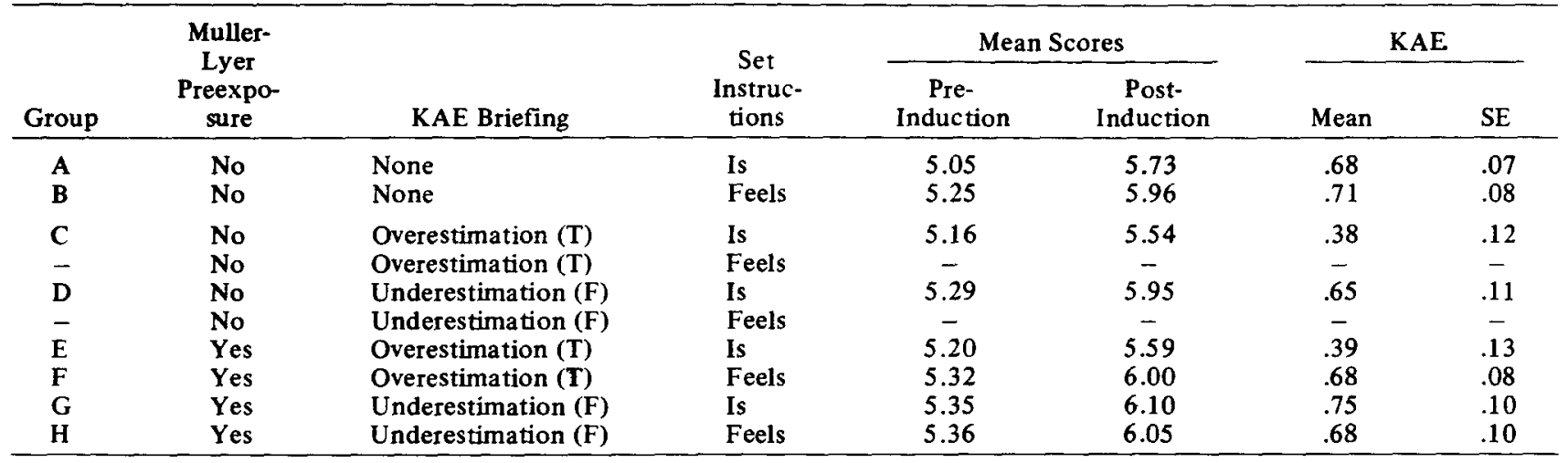

Note-Letters in parentheses indicate whether the briefing information was true (T) or false (F). The objective width of the $T$ block was $5.08 \mathrm{~cm}$.

illusion and $\mathrm{KAE}$, at its simplest. is the linking of directionality effects.

\section{Procedures for Groups}

Treatment variations among groups were of three kinds: (1) The verbal briefing did or did not include practice with the visual Mueller-Lyer display. (2) The verbal briefing provided no information, true information, or false information as to the direction of the aftereffect. (The visual display always reinforced the verbal information provided about the KAE.) (3) Following the briefing, Ss received either phenomenological-set or physicalistic-set instructions for judging the $T$ block in the KAE task. Regardless of preliminaries, the KAE procedure itself was administered in identical fashion to all Ss.

The groups are listed in Table 1. A decision was made in advance that, in view of the size of the experiment, the two least informative groups would not be run unless an analysis of the other eight showed it to be necessary. We reasoned that if, in making a phenomenological judgment, an $\mathrm{S}$ could ignore a prior briefing that included a visual aid, then a briefing without a visual aid should provide equivalent data.

Details about the instructions are necessary in an experiment concerned with instructional set. The $S s$ who received no KAE briefing, Groups A and B, were told only, "This rubbing of the blocks will affect future size judgments." No directionality information was given. The other six groups received directionality information: "The rubbing effect is as follows: The effect of rubbing the narrow left-hand block is to make the standard block feel wider (narrower). The effect of rubbing the wider right-hand block is to make the wedge seem narrower (wider). The overall effect is to cause you to overestimate (underestimate) the width of the standard block." The difference between instructions to the truth groups, C, E, and F, and falsehood groups, $D, G$, and $H$, was that the former received the italicized words, and the latter, the words in parentheses. The instructions for phenomenological and physicalistic set were identical to those employed by Wertheimer and Sheets, as quoted in our introduction, except that the word "block" was substituted for "bar."

\section{RESULTS}

The data from the Mueller-Lyer arrowhead displays (the visual aid) corresponded closely to that gathered by Wertheimer and Sheets. Compared with the phenomenological-set instructions, the physicalistic-set instructions shifted judgments of line length toward veridicality for every $S$. Groups $E$ and $F$ judged the upper display in Fig. 1. For these 48 Ss, phenomenological-set instructions led to a mean overestimation of $3.46 \mathrm{~cm}$ (standard error of the mean = $.15 \mathrm{~cm}$ ); the physicalistic-set instructions produced a mean overestimation of $.56 \mathrm{~cm}\left(\mathrm{SE}_{\overline{\mathrm{X}}}=.14 \mathrm{~cm}\right)$. Groups $G$ and $H$, judging the lower display of Fig. 1, gave a mean underestimation with a phenomenological set of $2.98 \mathrm{~cm}\left(\mathrm{SE}_{\overline{\mathrm{X}}}=.14 \mathrm{~cm}\right)$, with a physicalistic set of $1.30 \mathrm{~cm}\left(\mathrm{SE}_{\overline{\mathrm{X}}}=.09 \mathrm{~cm}\right)$. The mean difference between the two kinds of instructions was $2.90 \mathrm{~cm}$ (SE of the mean difference $=.15 \mathrm{~cm}$ ) for Groups $E$ and $F$, and 1.68 $\mathrm{cm}(\mathrm{SE}=.15 \mathrm{~cm})$ for Groups $\mathrm{G}$ and $\mathrm{H}$. Each of these six means exceeds the .0001 criterion level for a two-tailed $z$ test. Therefore, it can be stated that the physicalistic set reduced the measured magnitude of the Mueller-Lyer illusion, but not to zero.

The KAE data are presented in Table 1. Means of the preinduction and postinduction judgments are listed. The KAE score is obtained by subtracting preinduction from postinduction judgments. In conformity with the Wertheimer-Sheets data, the preinduction judgments across all but one group overestimate the size of the $5.08-\mathrm{cm}$ test block. This finding will not be commented upon further because we have no explanation to offer. For all groups, the postinduction mean increased, leading to considerable overestimation of the width of $T$. The KAE mean (difference) scores indicate the net overestimation. Two quantities are useful in evaluating these KAE scores: The average standard error of a $\mathrm{KAE}$ mean is $.10 \mathrm{~cm}$, and the average standard error of a difference (difference between mean KAE scores) is $.14 \mathrm{~cm}$. In terms of statistical significance, the $\mathrm{KAE}$ results indicate that all groups showed evidence of width contrast, i.e., all KAE scores are greater than zero, and two clusters of KAE scores exist, scores near .4 and $.7 \mathrm{~cm}$.

\section{DISCUSSION}

First and foremost, the data that resulted from a close 
replication of the Wertheimer-Sheets (1968) experiment confirm the data of that experiment in nearly all respects. The replication results include data for the visual Mueller-Lyer overestimation display (the truth display of Fig. 1) and KAE data for Groups E and F.

To proceed beyond the Wertheimer-Sheets replication, if $\mathrm{S}$ was asked to judge how the test block feels, then the nature of the briefing was irrelevant. No prior briefing, and a prior briefing reinforced by a visual aid (truthful or otherwise), gave equivalent data. Compare KAE scores for Groups B, F, and $\mathrm{H}$, which received the phenomenological-set instructions. The decision to omit the two phenomenological-set groups who were to receive a verbal briefing without a visual aid seems justified, since $S s$ can ignore a briefing plus visual aid.

When $\mathrm{S}$ was asked for a judgment about objective size, how wide $\mathrm{T}$ actually was, then prior KAE information was important. In the absence of prior information, the physicalistic judgments of Group A matched the phenomenological judgments of Group B. With truthful prior information, the measured size of the aftereffect was cut nearly in half (Groups $\mathrm{C}$ and $\mathrm{E}$ ). Note that the verbal briefing was sufficient; the visual aid added nothing. False prior information did not produce significant additional overestimation (Groups D and G), although the lie reinforced by Mueller-Lyer misinformation (Group G) lay in that direction. Therefore, the effective conditions for modifying the usual magnitude, $.7 \mathrm{~cm}$, of the aftereffect was a truthful verbal briefing followed by instructions to judge objective size. Practice and instruction with the visual Mueller-Lyer display were ineffective in modifying any KAE judgments.

Assume that Ss cooperate with E, listening to and attempting to abide by his explanations and instructions. The results indicate that Ss are not disregarding E's words, because KAE scores are not equal across groups. Let us propose that a briefing concerning KAE does not change a perceiver's experience of the width of the test block because he can and does disregard briefing information when asked how wide a block feels. Common sense tells us that we cannot transcend our own kinesthetic experience to return to the real object by will alone. Thus, the mere instruction to produce a judgment of objective width is a useless instruction by itself. The data show that KAE for Group B equals KAE for Group A in support of the argument. With a truthful prior briefing and a physicalistic set, Ss cannot null the aftereffect, bringing it to zero. Thus, a briefing concerning the directionality of effects does not provide sufficient information for making veridical judgments. The evidence is consistent with the position that the prior information provided in our experiment did not influence experienced width.

There is a paradox. The Ss responded to truthful prior information but not to falsified prior information. The fact that Groups A and B produced equal means implies that $\mathrm{Ss}$ in the physicalistic-set group had not gleaned the knowledge necessary to alter judgments toward veridicality through peeking, actions of $E$, self-experimentation, etc. But if $\mathrm{Ss}$ seem unable to discover anything on their own, then how are they able to reject false instructions? That is, why did not Ss in Groups $D$ and $G$ put their false information to work by inflating width judgments well above the usual $.7 \mathrm{~cm}$ ? We do not have a satisfactory answer. Perhaps, most Ss in these groups detected chicanery and acted like members of Group A (i.e., acted like misinformation was no information). Another possibility is that there exists a limit to overestimation along the wedge beyond which Ss are unwilling to widen their judgments. A natural limit is maximum span between the fingers, but the widths employed are far removed from that. In a previous experiment testing the cumulative impact of repeated inductions, an upper bound was reached early (Weintraub, Green, \& Herzog, in press). The first test of the limit hypothesis should be to rerun the present experiment, switching the wedge to the left hand, where underestimation would be the overall effect. A lower bound was not found after seven sessions per $S$ for underestimation along a wedge (Weintraub, Green, \& Herzog, in press).

The within-S data from the visual Mueller-Lyer display (Groups E, F, G, H) reinforce the $\mathrm{KAE}$ conclusions. Each $S$ served in both a phenomenological-set and a physicalistic-set condition, in that order. An S, by being permitted to see and discuss his own data generated under a phenomenological set and learning true line lengths, obtained a great deal of (truthful) information about how to null his own error. Note that, unlike KAE, the Mueller-Lyer arrowhead discussion provided $S$ with magnitude as well as directionality information. Although a large portion of the illusory effect was eliminated, a statistically significant Mueller-Lyer illusion remained when objective matches were then attempted. Precise information concerning his errors drew $S$ close to a veridical judgment, but not close enough.

In summary, Ss will cooperate by altering judgments toward veridicality when provided with truthful information about directionality. Ss will also cooperate by ignoring all prior information when asked to judge felt width. There was no transfer of training, so to speak, from a visual illusion to KAE, since the Mueller-Lyer practice did not increase the impact of a verbal briefing. We conclude that, for kinesthetic aftereffects, prior information can modify judgments under appropriate circumstances, but prior information does not modify felt width.

Regardless of interpretation, the experimental evidence buttresses a practical recommendation. The safest course, when investigating KAE itself, is to ask $S$ to judge how the test block feels. As long as $S$ has or obtains no information about the KAE task, there is no difference between judgments under a physicalistic or a phenomenological set. However. Ss may use what they 
know (or perhaps assume) if set instructions are either biased toward objective width or ambiguous. Ss seem willing and able, when asked, to disregard the hypotheses they hold, bringing under control one of the many sources of KAE variance. From the standpoint of an experimenter, the truly important helping hand belongs to a properly instructed, cooperative subject.

\section{REFERENCES}

Becker, W. C. Cortical inhibition and extraversion-introversion.
Journal of Abnormal \& Social Psychology, 1960, 61, 52-66. Weintraub, D. J., Green, G. S.. \& Herzog, T. R. Kinesthetic aftereffects day by day: Trends, task features, reliable individual differences. American Journal of Psychology, in press.

Wertheimer, M., \& Sheets, C. A., Jr. Effect of instructional set on kinesthetic figural aftereffects. Journal of Experimental Psychology, 1968, 77, 692-695.

(Received for publication February 12, 1973; revision received April 9, 1973.) 